\title{
Morphological and degenerative changes in a suspected post-traumatic bifid mandibular condyle
}

\author{
Jon Cornwall ${ }^{1}$, Lasitha Samarakoon ${ }^{2}$, Demonge J. Antony ${ }^{2}$, George J. Dias ${ }^{1}$ \\ ${ }^{1}$ Department of Anatomy, University of Otago, Dunedin, New Zealand \\ ${ }^{2}$ Department of Anatomy, University of Colombo, Colombo, Sri Lanka
}

\begin{abstract}
Bifid mandibular condyle is a rare anatomical anomaly with uncertain aetiology and pathogenesis. Theories for the development of this condition include bony alterations post-trauma, however little evidence exists to support this theory. Furthermore, the likely alterations to joint morphology over time as a result of this condition - including possible degenerative changes - have not been highlighted. We describe a case of bifid mandibular condyle attributed to a post-traumatic aetiology with associated degenerative changes in a male Sri Lankan skull of approximately 40 to 50 years of age. Fractures of the left parietal bone, zygomatic bone and damage to the orbit were observed in addition to a bifid left mandibular condyle that had anteromedial and posterolateral condyles and remodeling of the mandible consistent with a fracture of the mandibular neck. Two fossae were noted in the squamous part of the temporal bone that corresponded to the two condyles. Degenerative alterations to both joint surfaces included damage to the articular surface and osteophyte formation around the articular margins. This case provides circumstantial evidence to support the development of bifid mandibular condyles post-trauma with observations including longterm modifications to temporomandibular joint morphology leading to subsequent alterations in masticatory muscle activity, and the first evidence of degenerative change associated with this condition. Both outcomes will potentially result in a loss in temporomandibular joint function and are clinically relevant consequences of bifid mandibular condyle.
\end{abstract}

Keywords: arthritis; bifid mandibular condyle; degeneration; temporomandibular joint

Anatomy 2014;8:27-31, (c) 2014 TSACA

\section{Introduction}

Bifid mandibular condyle (BMC) is a rare anatomical anomaly of unknown etiology and unclear pathogenesis that is described both in clinical and anthropological literature. ${ }^{[1-3]} \mathrm{BMC}$ is asymptomatic in many patients, often being detected on routine dental radiography, ${ }^{[3-5]}$ however some individuals with BMC deformity suffer difficulties with occlusion and mastication that may require surgical intervention to allow restoration of temporomandibular function. ${ }^{[6,7]}$ Bilateral BMC, although rare, has been reported, ${ }^{[7-12]}$ and several cases of BMC associated with temporomandibular joint ankylosis have been published. ${ }^{[13-15]}$ There does not appear to be any racial or gender predilection ${ }^{[4]}$ although there appears to be interracial variations in prevalence. $^{[5,16]}$
Two popular theories exist for BMC development. It has been suggested that BMC can be a consequence of a dysplasic process of the condyle cartilage at the early stages of its development, or that BMC can also be caused by trauma via bone remodeling after articular trauma or repeated micro-trauma from malocclusion. ${ }^{[17]}$ To support the theory of dysplasia, a developmental anomaly such as an embryopathy by a teratogenic agent has been suggested as a possible etiology where BMC exists without history of trauma and with no apparent link with the patients medical history ${ }^{[18,19]}$ A history of trauma to the mandibular condyle has been suggested as a cause for $25 \%$ of all BMC cases, ${ }^{[20]}$ while BMC supposedly resulting from a sagittal condylar fracture has only been reported once. ${ }^{[2]}$ However, even a clear history of trauma does not mean that the bifid 
condyle has been caused directly as a result of the trauma: a BMC with a history of trauma was evaluated using histology of the excised specimen and revealed evidence supporting congenital etiology instead of trauma. ${ }^{[22]}$ Similarly, other reports of trauma leading to bilateral BMC suggest that trauma can be more coincidental than causative. ${ }^{[]]}$

Previous case reports have therefore supported both the congenital dysplasic theory and demonstrate that in some cases the actual aetiology is unclear. Furthermore, despite the publication of cases supporting the theory of $\mathrm{BMC}$ arising from trauma, few reports that have provided reasonable evidence for this mechanism ${ }^{[2]}$ contributing to the pathogenesis of BMC. In addition, there are no reports of the potential articular or degenerative effects of long-term BMC, ${ }^{[23]}$ an important consideration when creating biomechanical models involving $\mathrm{BMC}$ and when considering the long-term consequences of leaving BMC untreated. Here we report on a skull with unilateral BMC that includes circumstantial evidence to support the pathogenesis of BMC arising from trauma, and also highlights the possible consequences of degenerative changes that may occur from untreated BMC.

\section{Case Report}

A single skull with a unilateral BMC was identified in the collection of the Department of Anatomy, University of Colombo, Sri Lanka. The observations and measurements of this skull were performed in a manner that complied with local institutional ethical requirements. A detailed osteological study was conducted that included assessment of the general features of the skull, the temporomandibular joints, mandibular condyles, mandibular fossae and skull base. All evidence of trauma as well as the bony landmarks including left side temporomandibular joint and the mandibular condyles were measured and photographed, including a scale bar for reference, using a Canon Powershot G10 14.7 megapixel camera (Canon Inc., Tokyo, Japan).

\section{General observations}

The skull with a BMC was from a modern Sri Lankan male based on departmental records and confirmation by biological anthropologists using sex-specific morphological characteristics such as orbit shape, supraorbital ridge, zygomatic and temporal lines, and external occipital protuberance. The approximate age at death was assessed as being around 40 to 50 years.

The dentition was edentulous. Mandibular prognathism was noted as well as left sided anterolateral cross bite in the canine to first molar region (Figure 1). A partially healed fracture-displacement was noted $3 \mathrm{~mm}$ inferi-

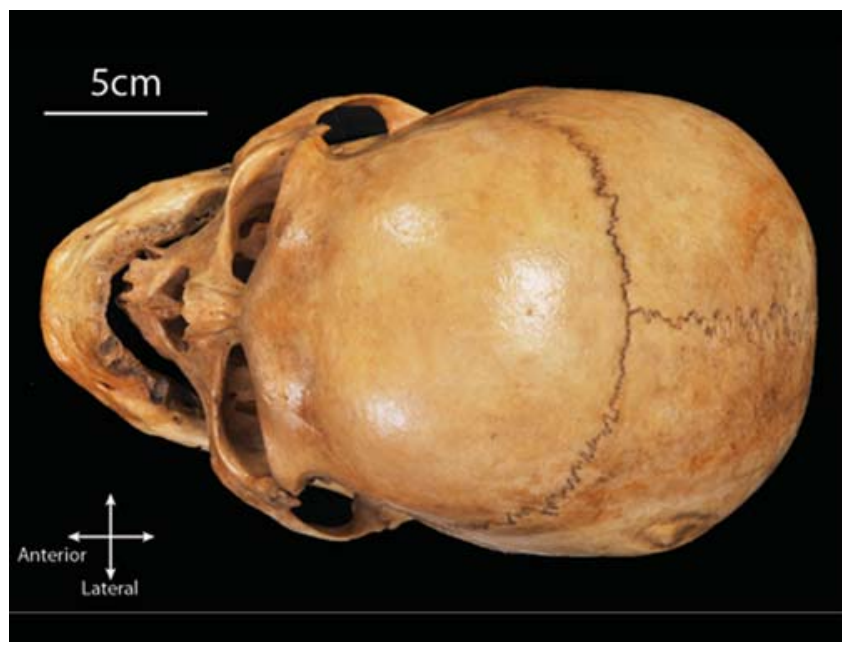

Figure 1. Superior view of skull and mandible demonstrating mandibular prognathism and left sided anterolateral cross bite.

or to the left fronto-zygomatic suture (Figure 2); displacement of the fracture with evidence of partial healing suggested the fracture was untreated and non-reduced (Figure 2). A healed depressed cranial vault fracture measuring $15 \mathrm{~mm}$ by $25 \mathrm{~mm}$ was also noted in the left mid parietal region, and a $1 \mathrm{~mm}$ by $4 \mathrm{~mm}$ sized bony fragment was missing in the anterolateral wall of the left orbit suggesting a unilateral level 3 Lefort fracture (Figure 2). It was unclear whether this was the result of an ante mortem injury or was a post mortem artifact.

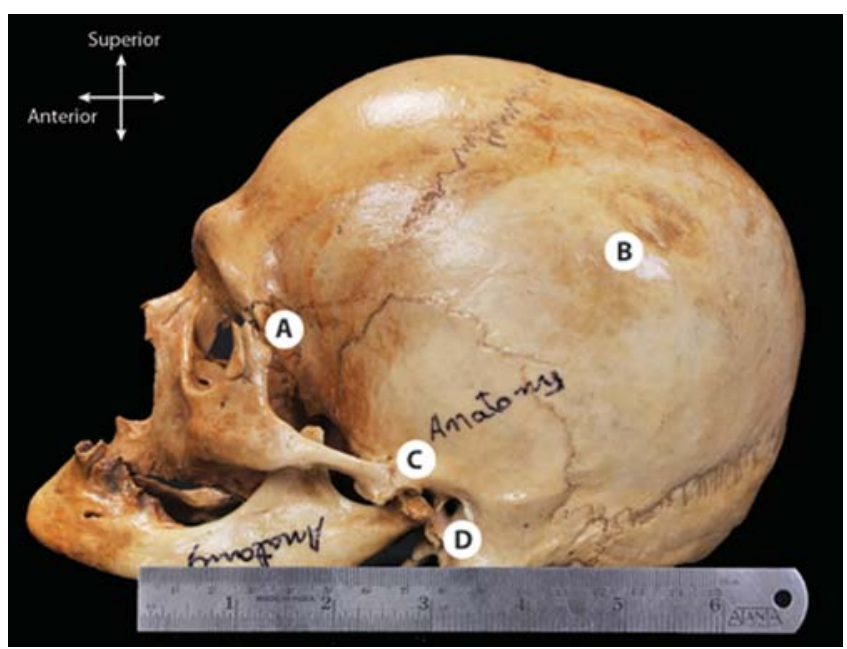

Figure 2. Lateral view of a partially healed and displaced fracture $3 \mathrm{~mm}$ inferior to the frontozygomatic suture on the left side $(\mathbf{A})$ as well as the depressed fracture of the left parietal region (B). Posterolateral secondary fossa notches the posterior end of the zygomatic arch (C). The groove between the posterior aspect of the two mandibular condyles butts the lateral tymphanic plate (D). 


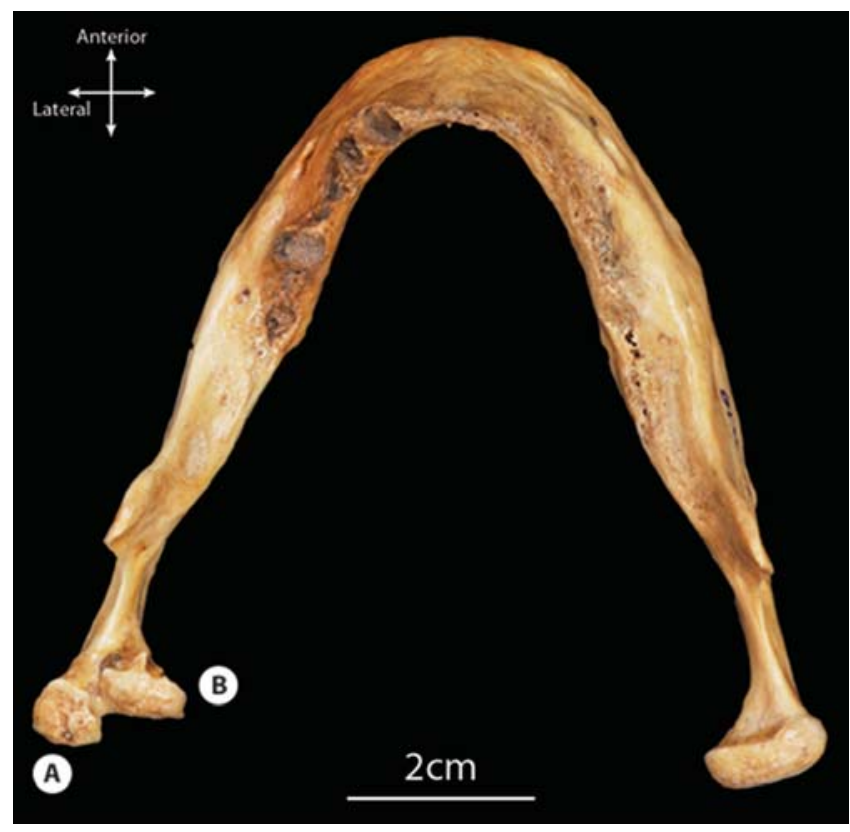

Figure 3. Superior view of the mandible showing the posterolateral (A) and anteromedial (B) condyles on the left part of the mandible with normal condyles on the right.

\section{Mandible}

An anteroposterior bifid condyle was noted on the left side (Figures 3 and 4). Two distinct condylar heads were separated by a $4 \mathrm{~mm}$ deep oblique sagittal groove and bony remodeling consistent with fracture of the mandibular neck. The groove between the condyles corresponded to the lateral lip of the tympanic plate of the temporal bone indicating that secondary condyle has formed lateral to the tympanic plate to accommodate condylar movement. On the medial condyle there was a distinct bony spur projecting in an anteromedial direction (Figures 3 and 4 ). This is compatible with the ossified attachment of the superior head of the lateral pterygoid muscle or an osteophyte.

Both condylar surfaces were rough with damage to the articular surface, sharp margins and osteophyte formation that were representative of arthritic change. The magnitude of the bony alterations was indicative of chronic osteoarthritis. No abnormalities were noted on the right mandibular condyle. On the left side the primary condyle was impinging on the posterior aspect of the mandibular notch; the mandibular notch on the right side was normal. No abnormalities were noted in the coronoid processes of either side, and the attachment sites of medial pterygoid muscle appeared normal on both sides. Gonial angles were unaffected bilaterally.

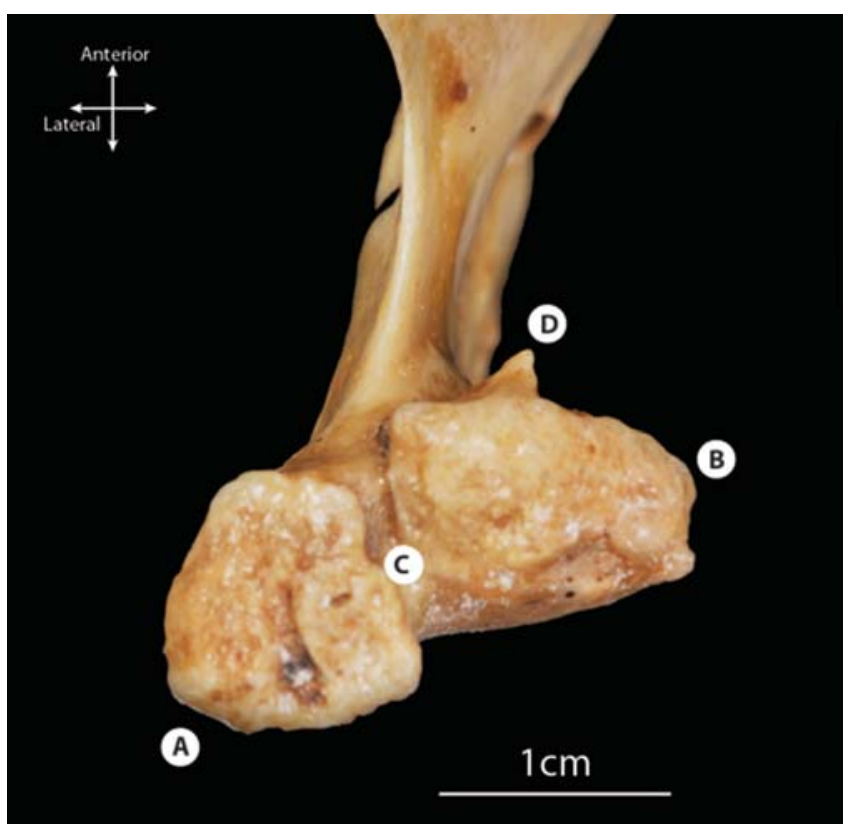

Figure 4. Superior and enlarged view of the bifid mandibular condyle. The posterolateral (secondary) condyle (A) is separated from the anteromedial (primary) condyle (B) by a deep sagittal groove (C). The bony spur on the primary condyle (D) is pointing in an anteromedial direction.

\section{Skull base}

Two distinct medial and lateral fossae, separated by a distinct ridge, were noted in the region of the left mandibular fossa (Figure 5). The lateral fossa was situated more posteriorly than the medial fossa. The morphology and anatomical location suggest that the pos-

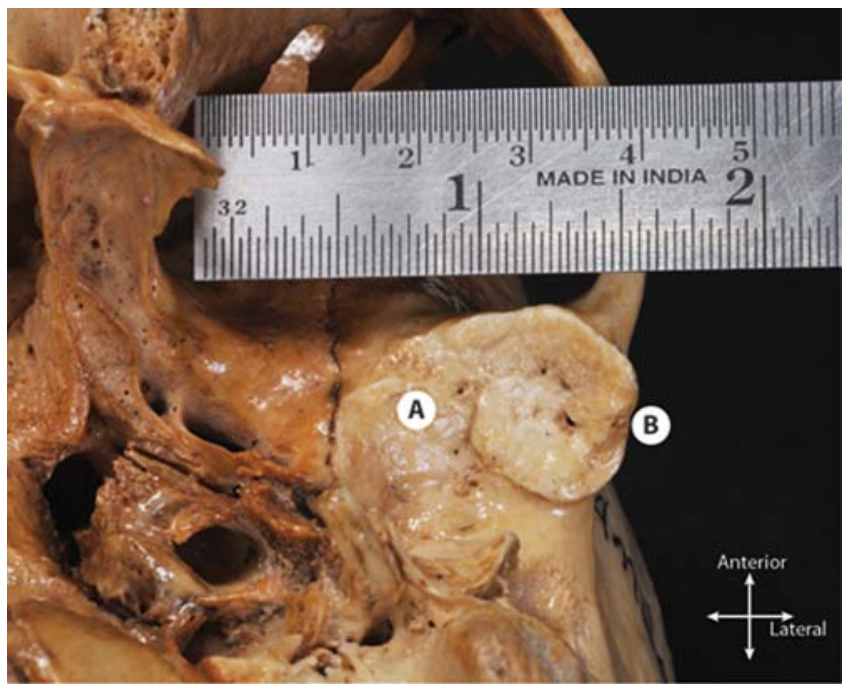

Figure 5. Photograph of the left mandibular fossa region showing two distinct fossae that are present in medial (A) and lateral (B) locations. 
terolateral fossa was the secondary fossa, with the anteromedial fossa being primary. When compared with the unaffected side, the antero-posterior length of the articular eminence was reduced by $8 \mathrm{~mm}$ on the affected side (12 $\mathrm{mm}$ on the right side, $4 \mathrm{~mm}$ on the left side). A bony prominence of the articular margins caused by the lateral lip of the secondary fossa was noted at the root of the left zygomatic arch. The anterior region of the infratemporal crest on the left side where the superior head of the lateral pterygoid muscle attaches was more prominent compared than the right. The lateral pterygoid plate was normal on both sides.

\section{Discussion}

This case study reports on a unilateral BMC in a single male skull of Sri Lankan origin, with circumstantial evidence supporting a possible theory of BMC development after trauma to the mandible by the presence of additional fractures to the cranium and frontozygomatic region of the facial skeleton, and the bony defect at the anterolateral wall of the orbit. Importantly, the case also highlights the likely sequelae of degenerative change, bony remodeling and potential chronic osteoarthritis that may occur as a result of untreated unilateral BMC. Information on degenerative bony changes in the articular surfaces of the joint secondary to BMC has not previously been reported and is of importance to clinicians as such information highlights likely long term morphological and functional alterations from untreated posttraumatic BMC.

\section{Fractures and bony alterations}

It is possible the initial trauma, responsible for all the visible fracture sites on the left side of the patients skull, lead to a fracture of the neck of the left mandible that resulted in the primary condyle being displaced anteromedially due to the tension of the lateral pterygoid muscle. The anterior region of the infratemporal crest was more prominent on the affected side, and it is possible that the altered direction of pull and unequal forces acting on the left temporomandibular joint lead to increased activity of the superior head of the lateral pterygoid muscle as evidenced by the prominent attachment site and bony spur (Figures 3 and 4). It is unclear whether the muscle would have hypertrophied and the increased forces lead to the bony alteration, or whether increased patterns of repeated activation would have contributed to such bony remodeling. Regardless, the function of the lateral pterygoid muscle on the affected side was likely abnormal thus contributing to the development of the bony spur and confirming that long term
BMC leads to alterations of masticatory muscle function. The observed cross bite may have been due to the posterior displacement of the secondary fossa (Figure 5) and the secondary condyle with regard to the contralateral mandibular fossa; such alterations are likely to have contributed to malocclusion and alterations in masticatory function.

\section{Degenerative change}

Closer inspection of both condylar surfaces and the left mandibular fossa margin revealed rough edges, damage to the articular surface and osteophyte formation that would support a diagnosis of chronic osteoarthritis (Figures 3-5). These degenerative changes were not present on the unaffected side. In order to accommodate the two mandibular condyles, the mandibular fossa on the affected side had undergone adaptation that gave rise to anteromedial and posterolateral fossae (Figure 5). A bony prominence caused by the lateral lip of the posterolateraly placed secondary fossa was also noted at the root of the left zygomatic arch. It also contributed to a left sided cross bite, however it is unclear if the BMC and resulting alteration of temporomandibular, occlusal and masticatory function contributed to the person's edentulous state. Degenerative change of this magnitude would likely affect the individual's temporomandibular function on a daily basis. Given the attachment of the temporomandibular joint capsule to the articular margins, it is likely that the BMC in this case caused some pain and temporomandibular joint dysfunction.

In bioanthropological literature the term bifid condyle often refers to pitting in the sagittal plain that divides the condyle mediolaterally, though it has been suggested that to avoid terminological ambiguity the term bifid condyle should only be used when describing multiple condyles in the sagittal plain as that this feature describes a true bifid condyle. ${ }^{[2]}$ In some instances it is unclear whether reported cases of BMC are a naturally occurring variation of intercondylar fissure depth as opposed to true BMC. ${ }^{[20,23,24]}$

\section{Limitations}

The assessment and inferences of this case of $\mathrm{BMC}$ being related to trauma is based on the assumption that the observed injuries to the left side of the skull and zygomatic region were related to the injury to the mandible. In the event that the observed BMC was unrelated to the trauma, this report still provides valuable information including previously unreported observations on degenerative changes that may arise as a result of unilateral BMC. 


\section{Conclusion}

This case demonstrates an instance of 'true' BMC with evidence that potentially supports post-traumatic aetiology, as well as highlighting a pattern of degenerative change and alteration in masticatory muscle function that may occur as a result of unilateral BMC. This important finding provides some indication of the potential type and severity of joint dysfunction that could occur if clinical intervention is not undertaken. Further osteoarcheological and clinical studies are required to provide further information about the causes and longterm outcomes in this rare condition.

\section{Acknowledgements}

This work was supported by the Otago School of Medical Sciences, University of Otago, and the Medical Faculty, University of Colombo. No financial contributions were involved.

\section{References}

1. Szentpetery A, Kocsis G, Marcsik A. The problem of the bifid mandibular condyle. J Oral Maxillofac Surg 1990;48:1254-7.

2. Dennison J, Mahoney P, Herbison P, Dias G. The false and the true bifid condyles. Homo 2008;59:149-59.

3. Lopez-Lopez J, Ayuso-Montero R, Salas EJ, Rosello-Llabres X. Bifid condyle: review of the literature of the last 10 years and report of two cases. Cranio 2010;28:136-40.

4. Loh FC, Yeo JF. Bifid mandibular condyle. Oral Surg Oral Med Oral Pathol 1990;69:24-7.

5. Menezes AV, de Moraes Ramos FM, de Vasconcelos-Filho JO, Kurita LM, de Almeida SM, Haiter-Neto F. The prevalence of bifid mandibular condyle detected in a Brazilian population. Dentomaxillofac Radiol 2008;37:220-3.

6. Gundlach KK, Fuhrmann A, Beckmann-Van der Ven G. The double-headed mandibular condyle. Oral Surg Oral Med Oral Pathol 1987;64:249-53.

7. Isik D, Sunay M, Bekerecioglu M. A case of bifid mandibular condyle causing mandibular dislocation. Eastern J Med 2011;16: 87-9.

8. Zohar Y, Laurian N. Bifid condyle of the mandible with associated polythelia and manual anomalies. J Laryngol Otol 1987;101: 1315-9.
9. McCormick SU, McCormick SA, Graves RW, Pifer RG. Bilateral bifid mandibular condyles. Report of three cases. Oral Surg Oral Med Oral Pathol 1989;68:555-7.

10. Stefanou EP, Fanourakis IG, Vlastos K, Katerelou J. Bilateral bifid mandibular condyles. Report of four cases. Dentomaxillofac Radiol 1998;27:186-8.

11. Alpaslan S, Ozbek M, Hersek N, Kanli A, Avcu N, Firat M. Bilateral bifid mandibular condyle. Dentomaxillofac Radiol 2004; 33:274-7.

12. Antoniades K, Hadjipetrou L, Antoniades V, Paraskevopoulos K. Bilateral bifid mandibular condyle. Oral Surg Oral Med Oral Pathol Oral Radiol Endod 2004;97:535-8.

13. To EW. Mandibular ankylosis associated with a bifid condyle. J Craniomaxillofac Surg 1989;17:326-8.

14. Daniels JS, Ali I. Post-traumatic bifid condyle associated with temporomandibular joint ankylosis: report of a case and review of the literature. Oral Surg Oral Med Oral Pathol Oral Radiol Endod 2005;99:682-8.

15. Sales MAO, Oliveira JX, Cavalcanti MGP. Computed tomography imaging findings of simultaneous bifid mandibular condyle and temperomandibular joint ankylosis: case report. Braz Dent J 2007; 18:74-7.

16. Miloglu O, Yalcin E, Buyukkurt M, Yilmaz A, Harorli A. The frequency of bifid mandibular condyle in a Turkish patient population. Dentomaxillofac Radiol 2010;39:42-6.

17. Ghigi G, Pastremoli A, Giuliani-Piccari G, Ruggeri F. Anatomic and radiological observations of the bifid mandibular condyle. Radiol Med 2001;101:152-6.

18. Cowan DF, Ferguson MM. Bifid mandibular condyle. Dentomaxillofac Radiol 1997;26:70-3.

19. Jordana X, Garcia C, Palacios M, Chimenos E, Malgosa A. Bifid mandibular condyle: archaeological case report of a rare anomaly. Dentomaxillofac Radiol 2004;33:278-81.

20. Li Z, Djae KA, Li Z-B. Post-traumatic bifid condyle: the pathogenesis analysis. Dent Traumatol 2011;27:453-4.

21. Antoniades K, Karakasis D, Elephtheriades J. Bifid mandibular condyle resulting from a sagittal fracture of the condylar head. $\mathrm{Br}$ J Oral Maxillofac Surg 1993;31:124-6.

22. Tunçbilek G. Bifid mandibular condyle: a rare disorder. J Craniofac Surg 2006;17:1207-9.

23. Acikgoz A. Bilateral bifid mandibular condyle: a case report. J Oral Rehab 2006;33:784-7.

24. Ramos FMM, Filho JOV, Manzi FR, Boscolo FN, Almeida SM. Bifid mandibular condyle: a case report. J Oral Sci 2006;48:35-7.

Correspondence to: George J. Dias, MS, PhD Department of Anatomy, University of Otago, POBox 56, Dunedin 9013, New Zealand

Phone: +64.3.479.7092 Fax: +64.3.479.7254

e-mail: george.dias@otago.ac.nz

Conflict of interest statement: No conflicts declared. 